\title{
Review on localized boundary integral equation: Discrete wavenumber method for 2D irregular layers*
}

\author{
Hong Zhou ${ }^{1, *}$ Xiaofei Chen ${ }^{2}$ and Ying Chang ${ }^{1}$ \\ ${ }^{1}$ Institute of Geophysics, China Earthquake Administration, Beijing 100081, China \\ ${ }^{2}$ School of Earth and Space Science, University of Science and Technology of China, Hefei 230026, China
}

\begin{abstract}
The pioneer study of simulating the wave field in media with irregular interface belongs to Aki and Larner. Since that many numerical methods on the subject have been developed, such as pure numerical techniques, ray method and boundary method. The boundary method based on boundary integral equation is a semi-analytical method which is suitable to modeling wave field induced by irregular border. According to the property of the applied Green's function the boundary methods can be sorted into space domain boundary method and wavenumber domain boundary method. For both of them it is necessary to solve a large equation, which means much computation is needed. Thus, it is difficult for the boundary methods to be applied in simulating wave field with high frequency or in large range. To develop a new method with less computation is meaningful. For this purpose, localized boundary integral equation, i.e., discrete wavenumber method is proposed. It is rooted in the Bouchon-Campillo method, an important wavenumber domain boundary method. Firstly the force on interface is separated into two parts: one is on flat part and the other on irregular part of the interface. Then Fourier transform is applied to identify their relation, the unknown distributes only on irregular part. Consequently computation efficiency is dramatically improved. Importantly its accuracy is the same as that of Bouchon-Campillo.
\end{abstract}

Key words: Bouchon-Campillo; irregular interface topography; Fourier transform; loBIE-DWM CLC number: P315.9 Document code: A

\section{Introduction}

Simulating the wave field in media with irregular interface is to an important issue in seismology. For this issue, pioneering work should be attributed to Aki and Larner (1970). They proposed a fast method with an assumption of Rayleigh Anta. But it only fits to simulating wave field due to slow dip interface (Cao et al, 2004). This method was also extended to calculate the irregular topography of the P-SV scattering wave field (Bouchon, 1973, 1976, 1979; Bouchon and Aki, 1977; Bard and Bouchon, 1980a, b, 1985) and irregular interface scattering two-dimensional P-SV wave field (Kohketsu, 1987; Kohketsu et al, 1991; Takenaka et al, 1996a), as well as the 3D wave field scattered irregular interface (Horike et al, 1990; Uebayashi et al, 1992). Using wave function expansion method, Trifunac (1971,

\footnotetext{
* Received 30 October 2009; accepted in revised form 1 December 2009; published 10 February 2010.

* Corresponding author. e-mail: zhouhong@cea-igp.ac.cn

(C) The Seismological Society of China and Springer-Verlag Berlin Heidelberg 2010
}

1973) gave the two-dimensional semi-circular canyon SH wave field analytical solution, which became an important theoretical test tool for a variety of SH-wave field numerical simulation methods. Wave function expansion method can also present the two-dimensional P-SV scattering wave field analytical solution with the arc, semi-circular or circular interface (Lee and Karl, 1992, 1993; Moeen-Vaziri and Trifunac, 1988, Liang et al, 2001).

Apart from the above approaches, other two categories of method, pure numerical simulation method (such as finite element, finite difference, etc.) and boundary integral equation method, were also important. Pure numerical simulation method including finite element (Smith, 1975; Koketsu et al, 2004), finite difference (Boore, 1970, 1972; Boore et al, 1971; Drake, 1972; Fuyuki and Matsumoto, 1980; IDlan, 1977; Graves, 1996; Levander, 1998; Moczo, 1989; Coutant et al, 1995), pseudo spectral method (Kolsloff and Baysal, 1982, Kolsloff et al, 1984; Furumura et al, 1998; Wang et al, 2001), spectral element method (Komatitsch, 2000; 
Komatitsch and Tromp, 2002; Komatitsch and Vilotte, $1998)$ etc. They are applicable to all kinds of complicated geological structure of the wave field, but for a limited computational domain, wave field on its borders must be specially handled, such as adding a variety of absorbing boundary conditions, otherwise, their results would be affected by the artificial border.

The boundary method, which was applied to calculating the irregular topography of the SH-wave scattering problems by Wong and Jenning (1975) for the first time based on Banaugh and Goldsmith (1963) formula, has been greatly developed. Green's function is the nuclear of the boundary integral equation, which can be represented in space domain or wave number domain. Spatial domain form is complex (Aki and Richards, 1980) and has singularity, while the wave number domain form is simple and has no singularity. Thus it is widely used in seismic exploration, micro-earthquakes, source inversion, fault rupture, stress calculation and seismic wave propagation simulation in $2 \mathrm{D} / 3 \mathrm{D}$ media. Based on different Green's function the boundary method can be sorted into spatial method (Sanchez-Sesma and Campillo, 1991, 1993; Sanchez-Sesma and Luzon, 1995; Gil-Zepeda et al, 2002) and wave number method (Bouchon, 1979, 1985, 1989, 1996a, b; Campillo and Bouchon, 1985; Gaffet and Bouchon, 1989; Kawase, 1988; Kawase and Aki, 1989, 1990; Chen, 1990, 1995, 1999; Cao, 2003; Cao et al, 2004; Takenaka, 1996b).

This boundary method usually involves solving a large full vector of linear equations, so it needs a large quantity of calculation. How to reduce the calculation of volume is the key to make it more practical method in use. Kawase (1988), Kawase and Aki (1989) (also Gaffet and Bouchon, 1991) used wave number domain half-space Green's function to limit the displacement and stress within the undulating surface, thus significantly reducing the number of unknowns. Unfortunately, for the uplift of the terrain, half-space Green's function is non-existent. This method must be plus a virtual interface within the uplift region which makes the problem to be a complex layer problem. Furthermore the half-space Green's function only fit to half-space problem. Campillo (1987) used layered Green's function to simulate underground drifts scattered wave field, and the layered media Green's function limited the unknown quantity to irregular at the interface. But there is no simple mathematical expression of layered Green's function, which needs special calculation, so that efficiency is not particularly evident. Bouchon (1996a, b) made the coefficient matrix to bandwidth by setting the unknown coefficient variable to zero which is less than a threshold value. However, this treatment reduces the calculation accuracy (Yokio and Sanchez-Sesma, 1998). We must be careful to apply it. On the basis of Bouchon-Campillo discrete wave number method, we proposed a different new approach, localized boundary integral equation, i.e., discrete wave number method (loBIE-DWM), while retaining the high precision under the premise of improving computational efficiency.

\section{Thought ofloBIE-DWM}

The discrete wave number domain boundary method, no matter how specific operations, must include the handling of the cyclical and imaginary frequency, which are the two most basic elements of the generalized discrete wave number method.

\subsection{Introduction of the calculation of cycle length $L$}

Figure 1 shows a medium with a line power source. Displacement and stress of excitation source in the medium can be expressed as follows:

$$
\boldsymbol{G}(x, z, \omega)=\mathrm{e}^{\mathrm{i} \omega t} \int_{-\infty}^{+\infty} f(k, z) \mathrm{e}^{-\mathrm{i} k x} \mathrm{~d} k,
$$

where $k$ is wave number, $x=[-\infty,+\infty]$.

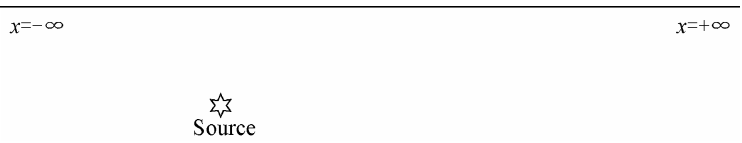

Figure 1 Original problem with line force source.

Figure 2 introduces the medium and source of the periodic distribution. Let $L$ the cycle length. One advantage of this approach is avoiding a singularity of $f(k, z)$. The second is to facilitate the numerical computation. When $L$ is long enough (Figure 2), the difference between the wave field due to cycle media and that due to the source with the original problem (Figure 1) can be ignored. It is clear from physical mechanism view that the greater $L$ is, the less the difference between them.

Introduction of the source of periodic distribution makes equation (1) become

$$
\boldsymbol{G}(x, z, \omega)=\mathrm{e}^{\mathrm{i} \omega t} \int_{-\infty}^{+\infty} f_{1}(k, z) \mathrm{e}^{-\mathrm{i} k x} \sum_{m=-\infty}^{m=+\infty} \mathrm{e}^{\mathrm{i} k m L} \mathrm{~d} k .
$$

Further simplified, truncate the discrete wavenum- 
ber $k$, the above equation become

$$
\boldsymbol{G}(x, z, \omega)=\frac{2 \pi}{L} \mathrm{e}^{\mathrm{i} \omega t} \sum_{n=-N}^{n=+N} f_{1}\left(k_{n}, z\right) \mathrm{e}^{-\mathrm{i} k_{n} x},
$$

where $k_{n}=\frac{2 \pi}{L} n$

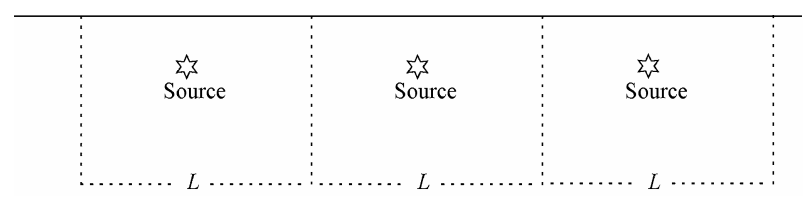

Figure 2 Change of the problem in Figure 1.

\subsection{Introduction of imaginary frequency $\omega_{I}$}

The introduction of cycle length $L$ makes the original problem with a single source become a problem with numbers of sources. If one can calculate the value of $G(x, z, \omega)$ with continuous frequency and use a continuous Fourier inverse transform to get non-periodic time response, based on the time difference between different sources, adding time window can separate out a single source of the wave field. But we can only have $G(x, z, \omega)$ on the discrete frequency $\omega=2 \pi / T$ ( $T$ is time window), and get solution in time domain through the discrete Fourier transform, imaginary frequency must be introduced to eliminate the impact of source in adjacent areas, namely:

$$
g(t, x, z)=\sum_{\omega_{R}=-M_{\omega}}^{M_{\omega}} G(x, z ; \omega) \mathrm{e}^{\mathrm{i} \omega_{R} t} \mathrm{e}^{\omega_{I} t} \mathrm{~d} \omega_{R},
$$

$\omega_{I}$ is a constant meeting

$$
\mathrm{e}^{\omega_{I} T}<<1
$$

and can make the signal outside the time window $T$ decay. So, $L$ can be selected long enough to ensure the time response within the time window $T$ being not subjected to interference from adjacent area sources.

Usually

$$
\omega_{I}=\left[-\frac{\pi}{T},-\frac{2 \pi}{T}\right] .
$$

It makes the signal attenuating in $\mathrm{e}^{\omega_{I} T}$ with time within the time windows.

\subsection{Theory ObBIE-DWM}

For topographical and multiple-layer problems the theory of $l o B I E-D W M$ is similar, though with different formulae. For simplicity here we only demonstrate its formula for the topography problem subjected to incident P-SV waves following the procedure in Zhou and Chen $(2008 \mathrm{a}, \mathrm{b})$. Other formulae can be seen in Zhou and Chen (2006a, 2009a).

\subsubsection{Formulation of BIE-DWM for P-SV topog- raphic problem}

The problem considered here is the seismic wave scattering due to an irregular topography subjected to incident P-SV waves in a homogeneous isotropic medium (Figure 3). In this problem, the total seismic wave field can be written as

$$
\left\{\begin{array}{c}
\boldsymbol{u}(x, z)=u_{\text {in }}(x, z)+u_{\mathrm{sc}}(x, z) \\
\boldsymbol{t}(x, z)=t_{\text {in }}(x, z)+t_{\mathrm{sc}}(x, z)
\end{array}\right.
$$

where $\boldsymbol{u}$ denotes the displacement in frequency domain, and the subscripts 'in' and 'sc' denote the incident and scattered wave fields, respectively. According to Huygens' Principle, the scattered waves can be represented by a Kirchhoff integral over the diffracting surface (see Bouchon, 1985, 1989; Campillo and Bouchon, 1985):

$$
\left\{\begin{array}{l}
u_{\mathrm{sc}}(x, z)=\int \boldsymbol{G}\left(x-x^{\prime}, z-z^{\prime}\right) \boldsymbol{q}\left(x^{\prime}, z^{\prime}\right) \mathrm{d} s \\
t_{\mathrm{sc}}(x, z)=\int \boldsymbol{H}\left(x-x^{\prime}, z-z^{\prime}\right) \boldsymbol{q}\left(x^{\prime}, z^{\prime}\right) \mathrm{d} s
\end{array}\right.
$$

where $\boldsymbol{G}$ is the tensor of Green's function for P-SV waves in a $2 \mathrm{D}$ elastic full space, $\boldsymbol{q}\left(x^{\prime}\right)$ is the distribution of the unknown force, and the integration domain $S$ is the irregular topographic surface. It is noted that the Green's tensor and force distribution are also the function of $\omega$, though it is not explicitly indicated here for compactness in mathematical presentation.

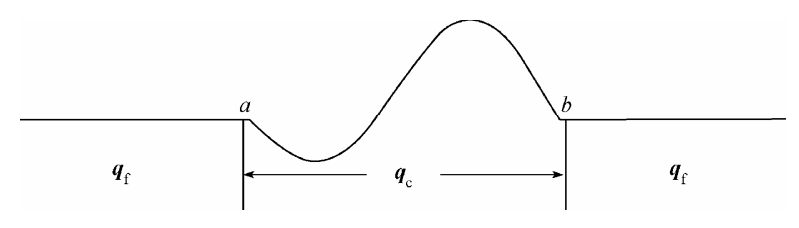

Figure 3 Ordinary topography model.

To solve this topographic scattering problem efficiently, we adopt the discrete wave number method (abbreviated as DWN) (see, e.g., Bouchon and Aki, 1977). With this method, the scattered wave field at an arbitrary discrete horizontal grid is reduced to the following finite summation over all the discrete Huygens source points (Bouchon, 1985, 1989; Compillo and Bouchon, 1985): 


$$
\left\{\begin{aligned}
u_{\mathrm{sc}}\left(x_{n}, z, \omega\right)= & \frac{L}{(2 N+1)} \cdot \\
& \sum_{m=-N}^{N} G^{\mathrm{DWN}}\left[x_{n}-x_{m}, z-\xi\left(x_{m}\right)\right] \cdot \boldsymbol{q}\left(x_{m}\right) \\
t_{\mathrm{sc}}\left(x_{n}, z, \omega\right)= & \frac{L}{(2 N+1)} \cdot \\
& \sum_{m=-N}^{N} H^{\mathrm{DWN}}\left[x_{n}-x_{m}, z-\xi\left(x_{m}\right)\right] \cdot \boldsymbol{q}\left(x_{m}\right),
\end{aligned}\right.
$$

where $\boldsymbol{G}^{\mathrm{DWN}}$ is the discrete wave number representation of Green's tensor $\boldsymbol{G},\left\{x_{m}, \xi\left(x_{m}\right) ; m=0, \pm 1, \pm 2, \ldots, \pm N\right\}$ are the locations of the Huygens point source along the irregular topographic surface, and $\boldsymbol{q}\left(x_{m}\right)$ denotes the strength of the $m$-th Huygens point source at the surface. $L$ is the length of periodicity of the source-medium configuration. $(2 N+1)$ is the total grids with an equal horizontal interval within one periodicity, thus $x_{m}=m \Delta x, m=0$, $\pm 1, \pm 2, \ldots, \pm N$ and $\Delta x=L /(2 N+1)$. Moreover, at the horizontal grids, Fourier transform of $\boldsymbol{G}^{\mathrm{DWN}}$ and its explicit expression is given in Appendix A.

The irregular surface is a free surface on which traction should vanish, i.e.,

$$
\begin{gathered}
t_{\text {in }}\left(x_{n}, z_{n}\right)+\sum_{m=-N}^{N}\left\{H^{\mathrm{DWN}}\left[x_{n}-x_{m}, z_{n}-\xi\left(x_{m}\right)\right]\right\}, \\
\boldsymbol{q}\left(x_{m}\right)=0,
\end{gathered}
$$

where $z_{n}=\xi\left(x_{n}\right)$ and $n=0, \pm 1, \pm 2, \ldots, \pm N \quad t_{\text {in }}$ and $H^{\mathrm{DWN}}$ are the tractions associated with $u_{\text {in }}$ and $G^{\mathrm{DWN}}$, respectively. Noticing that equation (8) provides exactly the number of linear equations as that of the unknowns $\boldsymbol{q}\left(x_{m}\right), m=0$, $\pm 1, \pm 2, \ldots, \pm N$, thus they could be directly applied to solving the unknowns after a minor modification on the collocation-elements of the coefficient matrix (Bouchon, 1985, 1989; Bouchon and Compillo, 1985). This is the basic idea of the BIE-DWM. Its validity and the applicability to practical problems have been demonstrated in a number of studies (Bouchon, 1985, 1989, 1996a, 1996b; Bouchon and Coutant, 1994; Campillo and Bouchon, 1985). It is obvious, however, that the computation cost of BIE-DWM directly depends on the total sampling number within one spatial periodicity, i.e., $x \in[-L / 2, L / 2]$. Because, on the one hand, the dimension of the coefficient matrix involved in this linear equation system is proportional to the total sampling number, while on the other hand, a proper estimation on the high frequency problem requires dense spatial sampling. Consequently, the dimension of the matrix is increased and so does the computation cost for high frequency problems.

\subsubsection{Formulation of the localized BIE-DWM for P-SV topographic problem}

Similar to the SH topographic problem (Zhou and Chen, 2006a), the first step for such an extension is an orthogonal decomposition of the unknown source strength vector $\boldsymbol{q}$, i.e.,

$$
\boldsymbol{q}=\boldsymbol{q}_{\mathrm{f}} \oplus \boldsymbol{q}_{\mathrm{c}},
$$

where, as shown in Figure $4, \boldsymbol{q}_{\mathrm{c}}$ denotes the unknown source strengths of those sampling points within the corrugated part of the surface, while $\boldsymbol{q}_{\mathrm{f}}$ denotes the unknowns of the flat part of the surface.

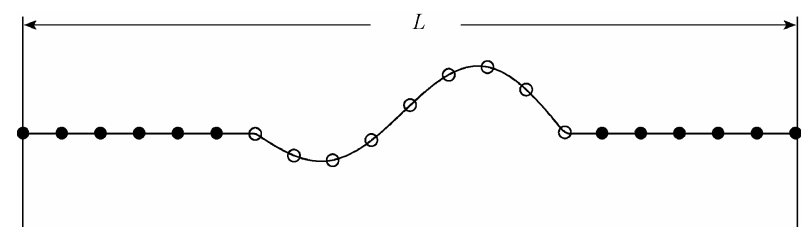

Figure 4 Cartoon of the orthogonal decomposition of the unknown source strength vector $\boldsymbol{q}$. Solid dots and open dots denote the unknown force source on the flat surface and those on the irregualr surface, respectively.

Substituting equation (9) into equation (8) yields

$$
\begin{gathered}
\sum_{m=-N}^{N} H^{\mathrm{DWN}}\left(x_{n}-x_{m}, 0\right)\left(x_{m}\right)+ \\
\sum_{m=N_{\mathrm{a}}}^{N_{\mathrm{b}}} H^{\mathrm{DWN}}\left[x_{n}-x_{m}, 0-\xi\left(x_{m}\right)\right] \boldsymbol{q}_{\mathrm{c}}\left(x_{m}\right)+t_{\mathrm{in}}\left(x_{n}, 0\right)=0,
\end{gathered}
$$

where $N_{\mathrm{a}}$ and $N_{\mathrm{b}}$ are the sampling point number corresponding to the irregular part of the topography in Figure 4. Note that equation (10) holds only for $x_{n} \in \Omega_{\mathrm{f}}$. For the range of $x_{n} \in \Omega_{\mathrm{c}}$ (in Figure 4), equation (10) does not hold any more, namely, the right-hand-side of equation (10) is equal to a non-zero quantity whose value is unknown yet at this stage. We denote this unknown quantity with $t_{\text {un }}\left(x_{n}\right)$, and it obviously has the property

$$
t_{\mathrm{un}}\left(x_{n}\right)=\left\{\begin{array}{cc}
\text { non - zero quanlity, } & x_{n} \in \Omega_{\mathrm{c}} \\
0, & x_{n} \in \Omega_{\mathrm{f}} .
\end{array}\right.
$$

With $\boldsymbol{t}_{\mathrm{un}}\left(x_{n}\right)$, equation (10) can be extended to the whole assembly of horizontal sampling within one spatial periodicity, i.e.,

$$
\begin{gathered}
\sum_{m=-N}^{N} H^{\mathrm{DWN}}\left(x_{n}-x_{m}, 0\right) \boldsymbol{q}_{\mathrm{f}}\left(x_{m}\right)+ \\
\sum_{m=N_{\mathrm{a}}}^{N_{\mathrm{b}}} H^{\mathrm{DWN}}\left(x_{n}-x_{m}, 0-z_{m}\right) \boldsymbol{q}_{\mathrm{c}}\left(x_{m}\right)= \\
-t_{\text {in }}\left(x_{n}, 0\right)+t_{\text {un }}\left(x_{n}\right),
\end{gathered}
$$


for $x_{n} \in[-L / 2, L / 2]$.

Then equation (12) can be executed by the discrete Fourier transform (DFT) with respect to $x_{n} \in[-L / 2, L / 2]$, and becomes

$$
\begin{gathered}
\tilde{\boldsymbol{H}}\left(k_{p}, 0\right) \widetilde{\boldsymbol{q}}_{\mathrm{f}}\left(k_{p}\right)+ \\
\sum_{m=N_{\mathrm{a}}}^{N_{\mathrm{b}}} \tilde{\boldsymbol{H}}\left(k_{p},-z_{m}\right) \boldsymbol{q}_{\mathrm{c}}\left(x_{m}\right) \exp \left(\mathrm{i} k_{p} x_{m}\right)= \\
-\widetilde{t}_{\text {in }}\left(k_{p}\right)+\frac{1}{2 N+1} \sum_{m=N_{\mathrm{a}}}^{N_{\mathrm{b}}} t_{\text {un }}\left(x_{m}\right) \exp \left(\mathrm{i} k_{p} x_{m}\right),
\end{gathered}
$$

where, $\tilde{\boldsymbol{H}}\left(k_{p}, z\right)$ is a DFT of $\left\{\tilde{\boldsymbol{H}}\left(x_{n}, z\right)\right\}$ and $\left\{\tilde{\boldsymbol{H}}\left(x_{n}, z\right)\right\}$ is given in Appendix A, and

$$
\begin{gathered}
\tilde{\boldsymbol{q}}_{\mathrm{f}}\left(k_{p}\right)=\frac{1}{2 N+1} \sum_{m=-N}^{N} \boldsymbol{q}_{\mathrm{f}}\left(x_{m}\right) \exp \left(\mathrm{i} k_{p} x_{m}\right), \\
\tilde{t}_{\text {in }}\left(k_{p}\right)=\frac{1}{2 N+1} \sum_{m=-N}^{N} t_{\text {in }}\left(x_{m}, 0\right) \exp \left(\mathrm{i} k_{p} x_{m}\right),
\end{gathered}
$$

with $k_{p}=2 \pi p / L$ for $p=0, \pm 1, \pm 2, \cdots, \pm N$.

Equation (13) can be solved as

$$
\begin{gathered}
\widetilde{\boldsymbol{q}}_{\mathrm{f}}\left(k_{p}\right)=-\left[\widetilde{\boldsymbol{H}}\left(k_{p}, 0\right)\right]^{-1} . \\
\left\{\sum_{m=N_{\mathrm{a}}}^{N_{\mathrm{b}}}\left[\tilde{\boldsymbol{H}}\left(k_{p},-z_{m}\right) \boldsymbol{q}_{\mathrm{c}}\left(x_{m}\right)-\frac{t_{\mathrm{un}}\left(x_{m}\right)}{2 N+1}\right] .\right. \\
\left.\exp \left(\mathrm{i} k_{p} x_{m}\right)+\tilde{t}_{\mathrm{in}}\left(k_{p}, 0\right)\right\} .
\end{gathered}
$$

Applying inverse DFT to equation (14) and exchanging summation order yields

$$
\begin{gathered}
\boldsymbol{q}_{\mathrm{f}}\left(x_{l}\right)= \\
-\sum_{n=N_{\mathrm{a}}}^{N_{\mathrm{b}}}\left\{\sum_{p=-N}^{N}\left[\widetilde{\boldsymbol{H}}_{0}\left(k_{p}\right)\right]^{-1} \widetilde{\boldsymbol{H}}\left(k_{p},-z_{n}\right) \exp \left[\mathrm{i} k_{p}\left(x_{n}-x_{l}\right)\right]\right\} . \\
\boldsymbol{q}_{\mathrm{c}}\left(x_{n}\right)-\sum_{p=-N}^{N}\left[\widetilde{\boldsymbol{H}}_{0}\left(k_{p}\right)\right]^{-1} \widetilde{t}_{\mathrm{in}}^{0}\left(k_{p}\right) \exp \left(-\mathrm{i} k_{p} x_{l}\right)+ \\
\sum_{m=N_{\mathrm{a}}}^{N_{\mathrm{b}}}\left\{\frac{1}{2 N+1} \sum_{p=-N}^{N}\left[\widetilde{\boldsymbol{H}}_{0}\left(k_{p}\right)\right]^{-1} \exp \left[-\mathrm{i} k_{p}\left(x_{l}-x_{m}\right)\right]\right\} \\
t_{u n}\left(x_{m}\right)
\end{gathered}
$$

for $x_{l} \in \Omega_{\mathrm{f}} ;$,and

$$
\begin{aligned}
0= & -\sum_{n=N_{\mathrm{a}}}^{N_{\mathrm{b}}}\left\{\sum_{p=-N}^{N}\left[\widetilde{\boldsymbol{H}}_{0}\left(k_{p}\right)\right]^{-1} \widetilde{\boldsymbol{H}}\left(k_{p},-z_{n}\right) \exp \left[\mathrm{i} k_{p}\left(x_{n}-x_{l}\right)\right]\right\} . \\
& \boldsymbol{q}_{\mathrm{c}}\left(x_{n}\right)-\sum_{p=-N}^{N}\left[\widetilde{\boldsymbol{H}}_{0}\left(k_{p}\right)\right]^{-1} \widetilde{t}_{\mathrm{in}}^{0}\left(k_{p}\right) \exp \left(-\mathrm{i} k_{p} x_{l}\right)+
\end{aligned}
$$

$$
\begin{gathered}
\sum_{m=N_{a}}^{N_{b}}\left\{\frac{1}{2 N+1} \sum_{p=-N}^{N}\left[\widetilde{\boldsymbol{H}}_{0}\left(k_{p}\right)\right]^{-1} \exp \left[-\mathrm{i} k_{p}\left(x_{l}-x_{m}\right)\right]\right\} . \\
t_{\text {un }}\left(x_{m}\right)
\end{gathered}
$$

for $x_{l} \in \Omega_{\mathrm{c}}$. Here, we denote $\widetilde{\boldsymbol{H}}_{0}\left(k_{p}\right)=\tilde{\boldsymbol{H}}\left(k_{p}, 0\right)$ and $\widetilde{t}_{\text {in }}^{0}\left(k_{p}\right)=\widetilde{t}_{\text {in }}\left(k_{p}, 0\right)$ for simplicity. In compact form, equations (15a) and (15b) are expressed as

$$
\begin{gathered}
\underline{\boldsymbol{Q}}_{\mathrm{f}}+\underline{\underline{\boldsymbol{A}}}_{\mathrm{f}} \underline{\boldsymbol{Q}}_{\mathrm{c}}=-\underline{\boldsymbol{S}}_{\mathrm{f}}+\underline{\underline{\boldsymbol{B}}}_{\mathrm{f}} \underline{\boldsymbol{T}}_{\mathrm{un}}, \\
\underline{\underline{\boldsymbol{A}}}_{\mathrm{c}} \underline{\boldsymbol{Q}}_{\mathrm{c}}=-\underline{\boldsymbol{S}}_{\mathrm{c}}+\underline{\underline{\boldsymbol{B}}}_{\mathrm{c}} \underline{\boldsymbol{T}}_{\mathrm{un}} .
\end{gathered}
$$

where $\underline{\underline{\boldsymbol{A}}}_{\mathrm{f}}, \underline{\underline{\boldsymbol{A}}}_{\mathrm{c}}, \underline{\underline{\boldsymbol{B}}}_{\mathrm{f}}, \underline{\boldsymbol{S}}_{\mathrm{f}}$ and $\underline{\boldsymbol{S}}_{\mathrm{c}}$ are the sub-blocks of matrices. $\underline{\underline{\boldsymbol{A}}}$ and $\underline{\underline{\boldsymbol{B}}}$, and vector $\underline{\boldsymbol{S}}$, are defined as:

$$
\begin{gathered}
\underline{\underline{A}}_{\mathrm{f}}=\left(\begin{array}{l}
\underline{\underline{A}}_{1} \\
\underline{\underline{A}}_{3}
\end{array}\right), \quad \underline{\underline{A}}_{\mathrm{c}}=\underline{\underline{A}}_{2}, \quad \underline{\underline{B}}_{\mathrm{f}}=\left(\begin{array}{l}
\underline{\underline{\boldsymbol{B}}}_{1} \\
\underline{\underline{\boldsymbol{B}}}_{3}
\end{array}\right), \\
\underline{\underline{\boldsymbol{B}}}_{\mathrm{c}}=\underline{\underline{\boldsymbol{B}}}_{2}, \quad \underline{\boldsymbol{S}}_{\mathrm{f}}=\left(\begin{array}{l}
\boldsymbol{S}_{1} \\
\underline{\boldsymbol{S}}_{3}
\end{array}\right), \quad \underline{\boldsymbol{S}}_{\mathrm{c}}=\underline{\underline{\boldsymbol{S}}}_{2} ;
\end{gathered}
$$

and

$$
\underline{\underline{\boldsymbol{A}}}=\left(\begin{array}{l}
\underline{\underline{\boldsymbol{A}}}_{1} \\
\underline{\underline{\boldsymbol{A}}}_{2} \\
\underline{\underline{\boldsymbol{A}}}_{3}
\end{array}\right), \underline{\underline{\boldsymbol{B}}}=\left(\begin{array}{l}
\underline{\underline{\boldsymbol{B}}}_{1} \\
\underline{\underline{\boldsymbol{B}}}_{2} \\
\underline{\underline{\boldsymbol{B}}}_{3}
\end{array}\right) \text { and } \underline{\boldsymbol{S}}=\left(\begin{array}{l}
\underline{\boldsymbol{S}}_{1} \\
\underline{\boldsymbol{S}}_{2} \\
\underline{\boldsymbol{S}}_{3}
\end{array}\right) \text {. }
$$

The dimensions of $\underline{\underline{\boldsymbol{A}}}$ and, $\underline{\underline{\boldsymbol{B}}}$ and vector $\underline{\boldsymbol{S}}$, are " $2(2 N+1) \times 2(2 N+1)$ " and " $2(2 N+1) \times 1$ ", and their block elements are defined by

$$
\begin{gathered}
(\underline{\underline{\boldsymbol{A}}})_{m n}=\sum_{p=-N}^{N}\left[\widetilde{\boldsymbol{H}}_{0}\left(k_{p}\right)\right]^{-1} \\
\widetilde{\boldsymbol{H}}\left(k_{p},-z_{n}\right) \exp \left[\mathrm{i} k_{p}\left(x_{n}-x_{m}\right)\right], \\
(\underline{\underline{\boldsymbol{B}}})_{m n}=\sum_{p=-N}^{N}\left[\widetilde{\boldsymbol{H}}_{0}\left(k_{p}\right)\right]^{-1} \exp \left[\mathrm{i} k_{p}\left(x_{n}-x_{m}\right)\right], \\
(\underline{\boldsymbol{S}})_{m}=\sum_{p=-N}^{N}\left[\widetilde{\boldsymbol{H}}_{0}\left(k_{p}\right)\right]^{-1} \widetilde{t}_{\mathrm{in}}^{0}\left(k_{p}\right) \exp \left(-\mathrm{i} k_{p} x_{m}\right),
\end{gathered}
$$

where $n, m=0, \pm 1, \pm 2, \ldots, \pm N$. The unknowns in equations (16a) and (16b) are

$$
\underline{\boldsymbol{T}}_{\mathrm{un}}=\left[\begin{array}{c}
\boldsymbol{t}_{\mathrm{un}}\left(x_{N_{\mathrm{a}}}\right) \\
\boldsymbol{t}_{\mathrm{un}}\left(x_{N_{\mathrm{a}}+1}\right) \\
\vdots \\
\boldsymbol{t}_{\mathrm{un}}\left(x_{N_{\mathrm{b}}}\right)
\end{array}\right], \underline{\boldsymbol{Q}}_{\mathrm{c}}=\left[\begin{array}{c}
\boldsymbol{q}_{\mathrm{c}}\left(x_{N_{\mathrm{a}}}\right) \\
\boldsymbol{q}_{\mathrm{c}}\left(x_{N_{\mathrm{a}}+1}\right) \\
\vdots \\
\boldsymbol{q}_{\mathrm{c}}\left(x_{N_{\mathrm{b}}}\right)
\end{array}\right], \quad \underline{\boldsymbol{g}}_{\mathrm{f}}=\left[\begin{array}{c}
\underline{\boldsymbol{Q}}_{\mathrm{f}, 1} \\
\underline{\boldsymbol{Q}}_{\mathrm{f}, 2}
\end{array}\right],
$$




$$
\underline{\boldsymbol{Q}}_{\mathrm{f}, 1}=\left[\begin{array}{c}
\boldsymbol{q}_{\mathrm{f}}\left(x_{-N}\right) \\
\boldsymbol{q}_{\mathrm{f}}\left(x_{1-N}\right) \\
\vdots \\
\boldsymbol{q}_{\mathrm{f}}\left(x_{N_{\mathrm{a}}-1}\right)
\end{array}\right] \text { and } \underline{\boldsymbol{g}}_{\mathrm{f}, 2}=\left[\begin{array}{c}
\boldsymbol{q}_{\mathrm{f}}\left(x_{N_{\mathrm{b}}+1}\right) \\
\boldsymbol{q}_{\mathrm{f}}\left(x_{N_{\mathrm{b}}+2}\right) \\
\vdots \\
\boldsymbol{q}_{\mathrm{f}}\left(x_{N}\right)
\end{array}\right]
$$

Combining equations (16a) and (16b) and eliminating the unknown vector $\underline{\boldsymbol{T}}_{\text {un }}$, we obtain a linear relationship between $\underline{\boldsymbol{Q}}_{\mathrm{f}}$ and $\underline{\boldsymbol{Q}}_{\mathrm{c}}$ as follows,

$$
\underline{\boldsymbol{Q}}_{\mathrm{f}}=-\underline{\boldsymbol{S}}_{\mathrm{f}}+\underline{\underline{\boldsymbol{B}}}_{\mathrm{f}}\left(\underline{\underline{\boldsymbol{B}}}_{\mathrm{c}}\right)^{-1} \underline{\boldsymbol{S}}_{\mathrm{c}}+\left\{\underline{\underline{\boldsymbol{B}}}_{\mathrm{f}}\left(\underline{\underline{\boldsymbol{B}}}_{\mathrm{c}}\right)^{-1} \underline{\underline{\boldsymbol{A}}}_{\mathrm{c}}-\underline{\underline{\boldsymbol{A}}}_{\mathrm{f}}\right\} \underline{\boldsymbol{Q}}_{\mathrm{c}} .
$$

To determine $\underline{\boldsymbol{Q}}_{\mathrm{c}}$, we recast equation (8) only on the corrugated part of the surface and obtain

$$
\underline{\underline{\boldsymbol{E}}}_{\mathrm{f}} \underline{\boldsymbol{Q}}_{\mathrm{f}}+\underline{\underline{\boldsymbol{E}}}_{\mathrm{c}} \underline{\boldsymbol{Q}}_{\mathrm{c}}=-\underline{\boldsymbol{T}}_{\mathrm{in}},
$$

where

$$
\left(\underline{\underline{E}}_{\mathrm{f}}\right)_{n m}=\boldsymbol{H}^{\mathrm{DWN}}\left[x_{n}-x_{m}, \xi\left(x_{n}\right)\right],
$$

for $x_{n} \in \Omega_{\mathrm{c}}$ and $x_{m} \in \Omega_{\mathrm{f}}$;

$$
\left(\underline{\underline{E}}_{\mathrm{c}}\right)_{n m}=\boldsymbol{H}^{\mathrm{DWN}}\left[x_{n}-x_{m}, \xi\left(x_{n}\right)-\xi\left(x_{m}\right)\right],
$$

for $x_{n}, x_{m} \in \Omega_{\mathrm{c}}$;

$$
\left(\underline{\boldsymbol{T}}_{\text {in }}\right)_{n}=t_{\text {in }}\left[x_{n}, \xi\left(x_{n}\right)\right],
$$

for $x_{n} \in \Omega_{\mathrm{c}}$.

Substituting equation (21) into (22) yields a linear equation system for determining the unknown $\underline{\boldsymbol{Q}}_{\mathrm{c}}$ as follows,

$$
\begin{gathered}
\left\{\underline{\underline{\boldsymbol{E}}}_{\mathrm{f}}\left[\underline{\underline{\boldsymbol{B}}}_{\mathrm{f}}\left(\underline{\underline{\boldsymbol{B}}}_{\mathrm{c}}\right)^{-1} \underline{\underline{\boldsymbol{A}}}_{\mathrm{c}}-\underline{\underline{\boldsymbol{A}}}_{\mathrm{f}}\right]+\underline{\underline{\boldsymbol{E}}}_{\mathrm{c}}\right\} \underline{\boldsymbol{Q}}_{\mathrm{c}}= \\
-\underline{\boldsymbol{T}}_{i n}+\underline{\underline{\boldsymbol{E}}}_{\mathrm{f}}\left[\underline{\boldsymbol{S}}_{\mathrm{f}}-\underline{\underline{\boldsymbol{B}}}_{\mathrm{f}}\left(\underline{\underline{\boldsymbol{B}}}_{\mathrm{c}}\right)^{-1} \underline{\boldsymbol{S}}_{\mathrm{c}}\right] .
\end{gathered}
$$

With this linear equation $\underline{\boldsymbol{Q}}_{\mathrm{c}}$ can be determined, thus $\underline{\boldsymbol{Q}}_{\mathrm{f}}$ can be consequently determined via equation (21). Finally, substituting the solved $\underline{\boldsymbol{Q}}_{\mathrm{c}}$ and $\underline{\boldsymbol{Q}}_{\mathrm{f}}$ into equation (6), we can calculate the P-SV wave field scattered by the irregular surface.

Compared with the BIE-DWM proposed by Bouchon and Campillo (1985, 1989), the loBIE-DWM for topographic problem of P-SV waves proposed here is more efficient in terms of computation. This is because, according to equation (23), the dimension of the matrices to be inverted in this method is proportional to the dimension of $\Omega_{\mathrm{c}}$ which is only a fraction of the di- mension of $\Omega_{L}$, while the dimension of the matrix to be inverted in BIE-DWM is proportional to the dimension of $\Omega_{L}$. Obviously, the more localized the corrugated part of the ground surface is, the more efficiently this new method performs.

The above description does not limit $\Omega_{\mathrm{c}}$ to a continuum region. This means the formulae are suitable to the topography with several separated irregular regions. In that case $\underline{\boldsymbol{Q}}_{\mathrm{c}}$ is composed of the total forces distributed on all irregular regions, while $\underline{\boldsymbol{Q}}_{\mathrm{f}}$ is composed of the total forces on all flat regions. For multiple-layer problem, the above theory is also suitable with different formulae (Zhou and Chen, 2009a, b).

\subsubsection{Validations of efficiency and accuracy}

In terms of accuracy, the results of the loBIE-DWM should be identical with those of BIE-DWM. Figure 5 displays our results (dot line) for the semi-cylindrical canyon's (with radius $a$ ) scattering problem from vertical plane $\mathrm{P}$ and $\mathrm{SV}$, showing identical results with those of BIE-DWM (solid line). The computational parameters used in these comparisons are: $L=20 a, \Delta x=0.2 a$ and $\eta=1$. Further, the loBIE-DWM performs more efficiently. Figure 6 shows the comparison of the computational times consumed by the two methods for this problem for taking different ratios of $L / a$ in the computation, where the $\Delta x / \mathrm{a}$ is fixed as 0.05 for all cases and the dimensionless frequency $\eta$ is set to be 1 . The computed results shown here were executed on a $\mathrm{PC} / \mathrm{P} 4$ with $2.8 \mathrm{GHz}$ clock speed. It is obvious that the loBIE-DWM becomes more efficient than the BIE-DWM as the ratio of $L / a$ increases, e.g., it is about 1000 times faster when $L / a=40$.

\section{Conclusions}

Bouchon-Campillo discrete wave number method (BC) avoids the singularity of Green's function in space domain, has high calculation precision, and can simulate the wave field of a number of geological formations. But it needs much calculation. For high-frequent or large-scale problems it is not competent. To find an alternative method with less calculation is deserved. To reduce the computation, we proposed an improved method (loBIE-DWM), of which the basic idea is to divide force source on the interface into two separate sections, use Fourier transform to decide their mathematical relationship, and then compose a linear equation only about force source on irregular part of interface. 

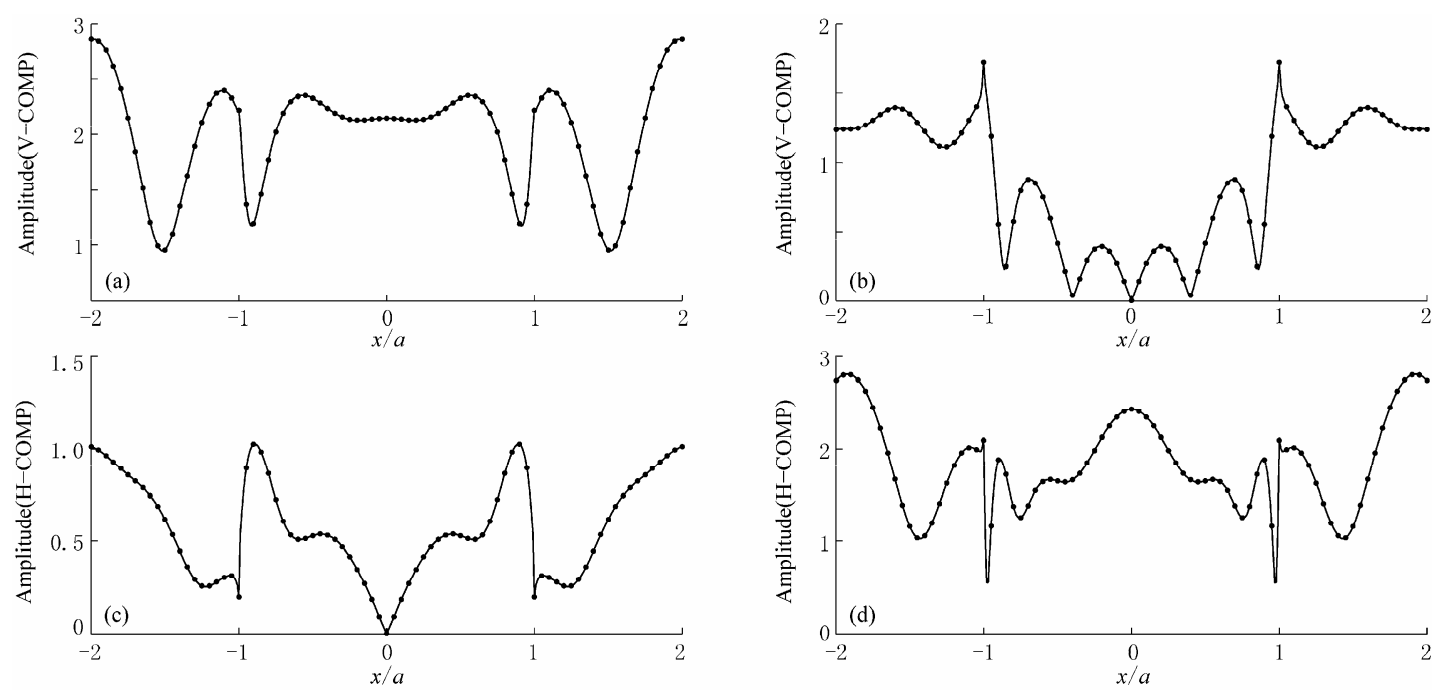

Figure 5 Comparison between our results and $\mathrm{BC}$ due to vertical plane $\mathrm{P}$ and $\mathrm{SV}$. The dot line is our results and the solid line is BC'results. (a) and (c) are respectively the vertical component and horizontal component due to incident P plane wave; (b) and (d) are the vertical component and horizontal component due to incident SV plane wave.

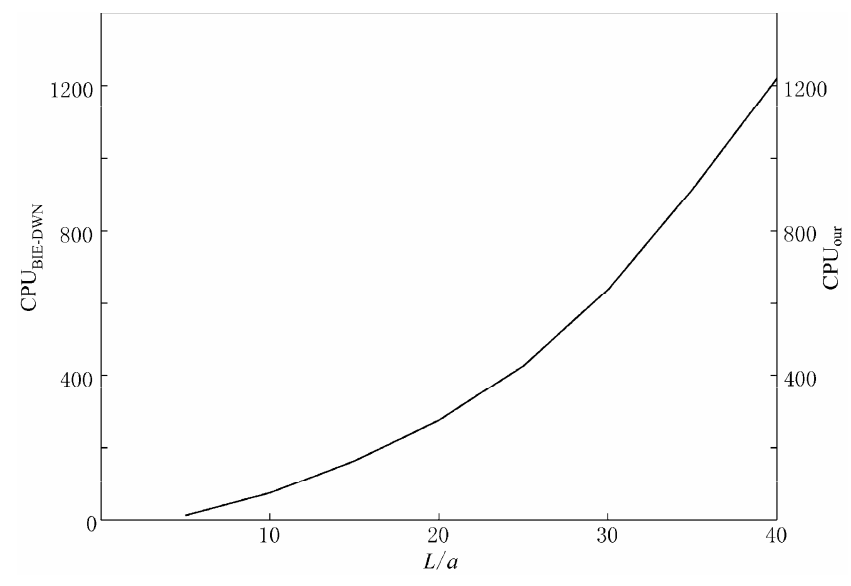

Figure 6 Ratio of CPU time between $\mathrm{BC}$ and our again $L / a$.

So it greatly reduces the computation and also can solve more problems. Its efficiency and valuation have been demonstrated in our studies (Zhou and Chen, 2006a, b, 2007, 2008 a, 2008 b, 2009a, b). With this method we can not only calculate the topography of the problem, but also solve the irregular interface issues. For the topography SH wave field calculations, the computation is only $1 / 10$ of that of $\mathrm{BC}$, while for the P-SV wave field the computation is only $1 / 100$ of that of BC. For the layered problem the advantage is clear. It is expected that the loBIE-DWM's advantage will be more remarkble with the progressing research.

Acknowledgements This study is jointly supported by National Natural Science Foundation of China (Nos.40874027, 90715020 and 90915012), IGPCEA
(DQJB07B06) and Special Public Welfare Industry (Nos.20070804 and 200808008).

\section{References}

Aki K and Larner K L (1970). Surface motion of a layered medium having an irregular interface due to incident plane $\mathrm{SH}$ wave. $J$ Geophys Res 70: 933-954.

Aki K and Richards P G (1980). Quantitative Seismology: Theory and Methods. W H Freeman \& Co., San Francisco, CA.

Banaugh R P and Goldsmith W (1963). Diffraction of steady acoustic waves by surface of arbitrary shape. J Acoust Soc Amer 35: 1590-1601

Bard P Y and Bouchon M (1980a). The seismic response of sediment-filled valleys: 1. The case of incident SH waves. Bull Seism Soc Amer 70: 1263-1286.

Bard P Y and Bouchon M (1980b). The seismic responses of sediment-filled valleys: 2. The case of incident P-waves and SV waves. Bull Seism Soc Amer 70: 1921-1 941.

Bard P Y and Bouchon M (1985). The two-dimensional resonance of sedimentary valley. Bull Seism Soc Amer 75: 519-541.

Boore D M (1970). Love waves in nonuniform wave guide: Finite difference calculation. J Geophys Res 75: 1 512-1 527.

Boore D M (1972). A note on the effect of simple topography on seismic SH wave. Bull Seism Soc Amer 62: 275-284.

Boore D M, Larner K L and Aki K (1971). Comparison of two independent method for the solution of wave scattering problem: Response of sedimentary basin to vertically incident SH waves. J Geophys Res 76: 558-569.

Bouchon M (1973). Effect of topography on surface motion. Bull Seism Soc Amer 63: 615-632.

Bouchon M (1976). Teleseismic body wave radiation from a seismic source in layered medium. Geophys J R astr Soc 47: 515-530.

Bouchon M (1979). Discrete wave number representation of elastic wave fields in three-space dimension. J Geophys Res 84: 3 609-3 614.

Bouchon M (1985). A simple, complete numerical solution to the problem of diffraction of SH waves by an irregular surface. J Acoust Soc Amer 77: 1-5.

Bouchon M (1989). A boundary integral equation-discrete wave-number representation method to study wave propagation in multilayered media having irregular interfaces. Geophysics 54: 1134-1 140.

Bouchon M (1996a). The discrete wave number formulation of boundary integral equations and boundary element methods: A review with applications to the 
simulation of seismic wave propagation in complex geological structures. Pure Appl Geophys 148(1-2): 3-20.

Bouchon M (1996b). Effect of three-dimensional topography on seismic motion. $J$ Geophys Res 101: 5835-5846.

Bouchon M and Aki K (1977). Discrete wavenumber representation of seismic source wave fields. Bull Seism Soc Amer 67: 259-277.

Bouchon M and Coutant O (1994). Calculation of synthetic seismograms in a laterally varying medium by the boundary element-discrete wavenumber method. Bull Seism Soc Amer 84: 1869 -1 881.

Campillo M (1987). Modeling of SH-wave propagation in an irregularly layered medium- application to seismic profiles near a dome. Geophys Prosp 35: 236-249.

Campillo M and Bouchon M (1985). Synthetic SH seismograms in a laterally varying medium by discrete wavenumber method. Geophys $J R$ astr Soc 83: $307-317$.

Cao J (2003). Seismogram synthesis for the 2D SH waves in irregularly multi-layered medium: Evaluations and applications. [Master Dissertation]. Peking University, 2003

Cao J, Ge Z X, Zhang J and Chen X F (2004). A comparative study on seismic wave methods for multi-layered media with irregular interfaces: Irregular topography problem. Chinese J Geophys 47(3): 495-503 (in Chinese with English abstract).

Chen X (1990). Seismograms synthesis for multi-layer media with irregular interfaces by global generalized reflection/transmission matrices method. Part I. Theory of 2-D SH case. Bull Seism Soc Amer 80: 1696-1 724.

Chen X (1995). Seismograms synthesis for multi-layer media with irregular interfaces by global generalized reflection/transmission matrices method. Part II . Application of 2-D SH case. Bull Seism Soc Amer 75: 1094-1 106.

Chen X (1999). Seismic wave propagation and excitation in multi-layered media with irregular interfaces Part I: SH case. Earthquake Research in China 13: $175-193$.

Coutant O, Virieux J and Zollo A (1995). Numerical source implementation in a 2D finite-difference scheme for wave propagation. Bull Seism Soc Amer 85: 1 507-1 512 .

Drake L L (1972). Love and Rayleigh waves in non-horizontal layered media. Bull Seism Soc Amer 62: 1241-1 258.

Furumura T, Kennett B L N and Furumura M (1998). Seismic wave field calculation for laterally heterogeneous whole Earth models using the pseudospectral method. Geophys J Int 135: 845-860.

Fuyuki M and Matsumoto Y. (1980). Finite difference analysis of Rayleigh wave scattering at a trench. Bull Seism Soc Amer 70: 2 051-2 069.

Gaffet S and Bouchon M (1989). Effect of two-dimensional topographies using the discrete wavenumber boundary integral equation method in P-SV case. $J$ Acoust Soc Amer 85: 2277-2 283.

Gaffet S and Bouchon M (1991). Source location and valley shape effects on the P-SV near displacement field using a boundary integral equation-discrete wave-number representation method. Geophys J Int 106: 341-355.

Gil-Zepeda S A, Luzon F, Aguirre J, Morales J, Sanchez-Sesma F J and Ortiz-Aleman C (2002). 3D seismic response of the deep basement structure of the Granada Basin (southern Spain). Bull Seism Soc Amer 92(6): 2 1632176.

Graves R W (1996). Simulating seismic wave propagation in 3D elastic media using staggered-grid finite-differences. Bull Seism Soc Amer 86: 1 0911107.

Horike M, Uebayashi $\mathrm{H}$ and Takeuchi Y (1990). Seismic response in three-dimensional sedimentary basin due to plane S-wave incidence. $J$ Phys Earth 38: 261-284.

IDlan A (1977). Finite difference modeling for P-pulse propagation in elastic media with polygonal surface. J Geophys 43: 41-58.

Kawase H (1988). Time-domain response of a semicircle canyon for incident SV, $\mathrm{P}$, and Rayleigh waves calculated by the discrete wave-number boundary element method. Bull Seism Soc Amer 78: 1415-1 437.

Kawase H and Aki K (1989). A study on the response of a soft basin for incident S, P, and Rayleigh waves with special reference to the long duration observed in Mexico City. Bull Seism Soc Amer 79: 1361-1 382.

Kawase H and Aki K (1990). Topography effect at the critical SV-wave incidence: Possible explanation of damage pattern by whittier Narrows, Cali- fornia earthquake of 1 October 1987. Bull Seism Soc Amer 80: 1-22

Kawase H and Aki K (1990). Topography effect at the critical SV-wave incidence: Possible explanation of damage pattern by whittier Narrows, California earthquake of 1 October 1987. Bull Seism Soc Amer 80: 1-22.

Kohketsu K (1987). 2-D reflectivity method and synthetic seismogram for irregularly layered structures: I. SH wave generation. Geophys $J R$ astr Soc 89: 821-838.

Kohketsu K, Kennett B L and Takenaka H (1991). 2-D reflectivity method and synthetic seismograms for irregularly layered structures: П. Invariant embedding approach. Geophys J Int 105: 119-130.

Koketsu K, Fujiwara H and Ikegamy Y (2004). Finite-element simulation of seismic ground motion with a voxel mesh. Pure Appl Geophy 161: 2183-2 198.

Komatitsch D and Vilotte J P (1998). The spectral element method: An efficient tool to simulate the seismic response of $2 \mathrm{D}$ and $3 \mathrm{D}$ geological structure. Bull Seism Soc Amer 88: 368-392.

Komatitsch D (2000). Simulation of anisotropic wave propagation based upon a spectral element method. Geophysics 65(4): 1 251-1260.

Komatitsch D and Tromp J (2002). Spectral-element simulations of global seismic wave propagation: I. Validation. Geophys J Int 149(2): 390-412.

Kosloff D and Baysal E (1982). Forward modeling by a Fourier method. Geophysics 47: $1401-1412$.

Kosloff D, Reshef M and Loewenthal D (1984). Elastic wave calculation by the Fourier method. Bull Seism Soc Amer 74: 875-891.

Lee V W and Karl J (1992). Diffraction of SV waves by underground circular cylindrical cavities. Soil Dynam Earthq Eng 11: 445-456.

Lee V W and Karl J (1993). On deformation near a circaular underground cavity subjected to incident plane P waves. European Journal of Earthquake Enigeering 7(1): 29-36.

Levander A R (1998). Fourth-order finite-diffrence P-SV seismograms. Geophysics 53: 1425-1 436.

Liang J W, Yan L J and Lee V W (2001). Scattering of plane P waves by circular-arc layered alluvial valleys: An analytical solution. Acta Seismologica Sinica 14(2): 176-195.

Moczo P (1989). Finite-diffrence technique for SH-wave in 2-D media using irregular grids: Application to the seismic response problem. Geophys $J$ Int 99: 321-329.

Moeen-Vaziri N and Trifunac M D (1988). Scattering and diffraction of plane P and SV-waves by two-dimensional inhomogeneities. Soil Dynam Earthq Eng 7: 189-200.

Sanchez-Sesma F J and Campillo M (1991). Diffraction of P, SV, and Rayleigh waves by topographic features: A boundary integral formulation. Bull Seism Soc Amer 81: 2234-2 253.

Sanchez-Sesma F J and Campillo M (1993). Topography effects for incident P, SV and Rayleigh waves. Tectonophysics 218: 113-125.

Sanchez-Sesma F J and Luzon F (1995). Seismic response of three dimensional alluvial valleys for incident P, S and Rayleigh waves. Bull Seism Soc Amer 85: $269-284$.

Smith W D (1975). The application of finite element analysis to body wave propagation problem. GeophysJ R astr Soc 42: 747-768.

Takenaka H, Kennett B L N and Fujiwa H (1996b). Effect of 2-D topography on the 3-D seismic wave field using a 2.5-D discrete wavenumber-boundary integral equation method. Geophys J Int 124: 741-755.

Takenaka H, Ohori M, Koketu K and Kennett B L (1996a). An efficient approach to the seismogram synthesis for a basin structure using propagation invariants. Bull Seism Soc Amer 86: 379-388.

Trifunac M D (1971). Surface motion of a semi-cylindrical alluvial valley for incident plane SH wave. Bull Seism Soc Amer 61: 1755-1 770.

Trifunac M D (1973). Scattering of plane SH waves by a semi-cylindrical canyon. Earthq Eng Struct Dynam 1: 267-281.

Uebayashi H, Horike M and Takeuchi Y (1992). Seismic motion in a three-dimensional arbitrarily-shaped sedimentary basin, due to a rectangular dislocation source. J Phys Earth 40: 223-240.

Wong H L, and Jennings P C (1975). Effect of canyon topographies on strong ground motion. Bull Seism Soc Amer 65: 1239-1 257.

Wang Y B, Takenaka H and Furumura T (2001). Modeling seismic wave propagation in a two-dimensional cylindrical whole-earth model using 
pseudo-spectral method. Geophys J Int 145: 689-708.

Yokoi T and Sanchez-Sesma F J (1998). A hybrid calculation technique of the indirect boundary element method and the analytical solutions for three-dimensional problems of topography. Geophys J Int 133(1): 121-139.

Zhou H and Chen X F (2006a). A new approach to simulate scattering of SH waves by an irregular topography. Geophys J Int 164(2): 449-459.

Zhou H and Chen X F (2006b). A study on frequency responses of topography with different scales due to incident SH wave. Chinese J Geophys 49(1): 205-211 (in Chinese).

Zhou H and Chen X F (2007). A study on the effect of depressed topography on Rayleigh surface wave. Chinese J Geophys 50(4): 1 182-1 189.

Zhou H and Chen X F (2008a). The simulation of strong ground motion in Beijing city due to Zhangbei earthquake. Progress in Geophysics 23(5): 1355-1 366 (in Chinese).
Zhou H and Chen X F (2008b). The localized boundary integral equation: Discrete wavenumber method for simulating P-SV wave scattering by an irregular topography. Bull Seism Soc Amer 98(1): 265-279.

Zhou H and Chen X F (2009a). The localized boundary integral equation-discrete wavenumber method for simulating wave propagation in irregular multiple layers. I: Theory. Bull Seism Soc Amer 99(3): 1 9841994.

Zhou H and Chen X F (2009b). The localized boundary integral equation-discrete wavenumber method for simulating wave propagation in irregular multiple layers II: Validation. Bull Seism Soc Amer 99(3): 19952011.

\section{Appendix A}

Green's function for displacement

$$
\boldsymbol{G}=\left[\begin{array}{cc}
G_{x x} & G_{x z} \\
G_{z x} & G_{z z}
\end{array}\right]
$$

where

$$
\begin{gathered}
G_{x x}=-\frac{\mathrm{i}}{2 L \mu} \sum_{n=-N}^{N}\left(\frac{k_{n}^{2}}{\gamma_{1}} \mathrm{e}^{-i \gamma_{1}\left|z-z_{c}\right|}+\gamma_{2} \mathrm{e}^{-i \gamma_{2}\left|z-z_{c}\right|}\right) \mathrm{e}^{-i k_{n}\left(x-x_{c}\right)} \\
G_{x z}=-\frac{\mathrm{i} \operatorname{sgn}\left(z-z_{c}\right)}{2 L \mu} \sum_{n=-N}^{N} k_{n}\left(\mathrm{e}^{-\mathrm{i} \gamma_{1}\left|z-z_{c}\right|}-\mathrm{e}^{-i \gamma_{2}\left|z-z_{c}\right|}\right) \mathrm{e}^{-i k_{n}\left(x-x_{c}\right)} \\
G_{z x}=G_{x z} \\
G_{z z}=-\frac{\mathrm{i}}{2 L \mu} \sum_{n=-N}^{N}\left(\gamma_{1} \mathrm{e}^{-\mathrm{i} \gamma_{1}\left|z-z_{c}\right|}+\frac{k_{n}^{2}}{\gamma_{2}} \gamma_{2} \mathrm{e}^{-\mathrm{i} \gamma_{2} \mid z-z_{c}}\right) \mathrm{e}^{-\mathrm{i} k_{n}\left(x-x_{c}\right)}
\end{gathered}
$$

\section{Appendix B}

Green's function for stress

$$
\boldsymbol{H}=\left[\begin{array}{ll}
H_{x x} & H_{x z} \\
H_{z x} & H_{z z}
\end{array}\right]
$$

where

$$
\begin{aligned}
& H_{x x}=\frac{1}{2 L k_{\beta}^{2}} \sum_{n=-N}^{N}\left\{\left[\frac{k_{n}}{\gamma_{1}}\left(2 \gamma_{1}^{n}-k_{\beta}^{2}\right) \mathrm{e}^{-\mathrm{i} \gamma_{1}\left|z-z_{c}\right|}-2 k_{n} \gamma_{2} \mathrm{e}^{-\mathrm{i} \gamma_{2}\left|z-z_{c}\right|}\right] n_{1}-\operatorname{sgn}\left(z-z_{c}\right)\left[2 k_{n}^{2} \mathrm{e}^{-\mathrm{i} \gamma_{1}\left|z-z_{c}\right|}+\left(2 \gamma_{2}^{2}-k_{\beta}^{2}\right) \mathrm{e}^{-\mathrm{i} \gamma_{2}\left|-z_{c}\right|}\right] n_{2}\right\} \mathrm{e}^{-\mathrm{i} k_{n}\left(x-x_{c}\right)}
\end{aligned}
$$

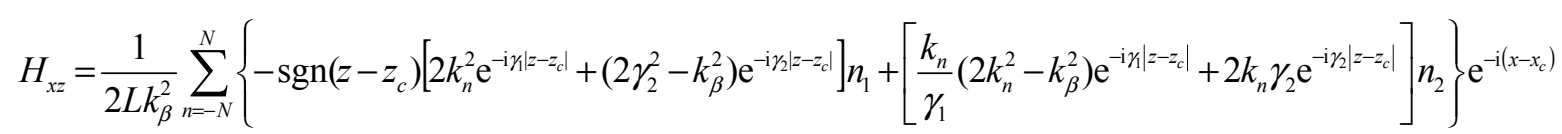

$$
\begin{aligned}
& H_{z x}=\frac{1}{2 L k_{\beta}^{2}} \sum_{n=-N}^{N}\left\{\operatorname{sgn}\left(z-z_{c}\right)\left[\left(2 \gamma_{2}^{2}-k_{\beta}^{2}\right) \mathrm{e}^{-\mathrm{i} \gamma\left|z-z_{d}\right|}+2 k_{n}^{2} \mathrm{e}^{-\mathrm{i} \gamma_{2} \mid-z_{d}}\right] n_{1}+\left[-2 k_{n} \gamma_{1} \mathrm{e}^{-\mathrm{i} \gamma\left|z-z_{c}\right|}+\frac{k_{n}}{\gamma_{1}}\left(2 \gamma_{2}^{2}-k_{n}^{2} \mathrm{e}^{-\mathrm{i} \gamma_{2}\left|z-z_{c}\right|}\right] n_{2}\right\} \mathrm{e}^{-i\left(x-x_{c}\right)}\right. \\
& H_{z z}=\frac{1}{2 L k_{\beta}^{2}} \sum_{n=-N}^{N}\left\{\left[-2 k_{n} \gamma_{1} \mathrm{e}^{-i \gamma_{1}\left|z-z_{c}\right|}+\frac{k_{n}}{\gamma_{2}}\left(2 \gamma_{2}^{2}-k_{\beta}^{2}\right) \mathrm{e}^{-\mathrm{i} \gamma_{2} \mid z-z_{c} c}\right] n_{1}+\operatorname{sgn}\left(z-z_{c}\right)\left[\left(2 k_{n}^{2}-k_{\beta}^{2}\right) \mathrm{e}^{-\mathrm{i} \gamma_{1}\left|z-z_{c}\right|}-2 k_{n}^{2} \mathrm{e}^{-\mathrm{i} \gamma_{2}\left|z-z_{c}\right|}\right] n_{2}\right\} \mathrm{e}^{-i k_{n}\left(x-x_{c}\right)}
\end{aligned}
$$

with $\gamma_{2}=\sqrt{\frac{\omega^{2}}{\beta^{2}}-k_{n}}, \operatorname{Im}\left(\gamma_{2}\right) \leq 0 ; \gamma_{1}=\sqrt{\frac{\omega^{2}}{\alpha^{2}}-k_{n}}, \operatorname{Im}\left(\gamma_{1}\right) \leq 0 ; \alpha$ and $\beta$ are the velocity of $\mathrm{P}$ and $\mathrm{S}$ waves, respectively. 